\title{
Comparison of methods for the detection of penicillinase-producing Neisseria gonorrhoeae
}

\author{
E H SNG,* K L YEO,* V S RAJAN, † AND A L LIM* \\ From the ${ }^{*}$ Department of Pathology, Outram Road, and †Middle Road Hospital, Singapore
}

SUMMARY In an evaluation of four methods for detecting penicillinase-producing Neisseria gonorrhoeae the chromogenic cephalosporin, rapid iodometric, and penicillin disc diffusion methods gave complete agreement for all the 202 strains of gonococci tested. No false-positive or false-negative results occurred. The filter paper iodometric method detected $99 \%$ of the penicillinase-producing strains without any false-positive result.

\section{Introduction}

The first report of penicillinase-producing Neisseria gonorrhoeae (PPNG) strains appeared in 1976. Since then a number of different tests have been described to detect such strains. These include the rapid iodometric method ${ }^{1}$ and its filter paper modifications, ${ }^{2}{ }^{3}$ the acidometric method ${ }^{4}$ and its microtitre modification, ${ }^{5}$ the chromogenic cephalosporin method, ${ }^{6}$ and various penicillin inhibition methods which use either the direct disc diffusion technique ${ }^{7}$ or indirect techniques using penicillin-sensitive Staphylococcus aureus ${ }^{8}$ or Sarcina lutea ${ }^{9}$ as indicator bacteria.

A number of these tests are currently in use in different laboratories. No large-scale study has so far been reported on the relative merits of the different methods. The present study has therefore been conducted to enable laboratories to decide which of the methods may be most suitable for their use.

We have compared the chromogenic cephalosporin, rapid iodometric (tube), filter paper iodometric, and penicillin disc diffusion methods. The acidometric method has not been included because preliminary experience indicated that results were sometimes not clear cut as the penicillin-phenol red reagent tended to turn acid when stored. The indirect techniques using penicillin-sensitive indicator bacteria were also excluded because the direct disc diffusion technique is simpler.

\section{Material and methods}

Freshly isolated $N$ gonorrhoeae strains were obtained from clinical specimens, which were inoculated

Address for reprints: Dr E H Sng, Department of Pathology, Outram Road, Singapore 0316, Republic of Singapore

Received for publication 8 January 1980 directly on to modified Thayer-Martin medium. Identification was made by colonial morphology, oxidase reaction, and Gram-stain microscopical examination.

All isolates were first tested for the production of penicillinase by the filter paper iodometric method. Penicillinase-positive isolates and an equivalent number of penicillinase-negative strains were then tested by a different staff member using the chromogenic cephalosporin, rapid iodometric, and disc diffusion methods.

\section{FILTER PAPER IODOMETRIC METHOD}

This is a modification of the technique by Odugbemi et $a l^{3}$. Pieces of Whatman No 1 filter paper, each measuring approximately $5 \times 6 \mathrm{~cm}$, were immersed in $1 \%$ soluble starch and dried. These may be stored at room temperature for one month. When needed, the starch paper was placed in a Petri dish and soaked with a solution containing $60000 \mu \mathrm{g} / \mathrm{ml}$ benzyl penicillin in phosphate-buffered saline, $\mathrm{pH} 7 \cdot 3$ (Oxoid). With a bacteriological loop, several colonies from a culture were spread over an area approximately $5 \mathrm{~mm}$ in diameter. Several different strains of gonococci may be tested on the same paper, separated from each other by approximately $1 \mathrm{~cm}$. The paper was incubated at $37^{\circ} \mathrm{C}$ for 30 minutes with the Petri dish cover on. After incubation the paper was soaked with a solution of iodine $1 \mathrm{~g}$, potassium iodine $2 \mathrm{~g}$ in distilled water $200 \mathrm{ml}$. The Petri dish was held at an angle of about $30^{\circ}$ to allow the iodine solution to drain to the bottom. The starch paper turned black within seconds. The spots where the PPNG strains were smeared remained colourless.

OTHER ASSAY METHODS

The methods for the rapid iodometric, chromogenic 
cephalosporin, and disc diffusion assays are as described by the WHO Scientific Group on Neisseria gonorrhoeae and gonococcal infections. ${ }^{10}$ The rapid iodometric assay was performed in test tubes. The organism was incubated with penicillin solution for 60 minutes instead of 30 minutes. A positive result was indicated by the decolorisation of the starch solution within 10 minutes. The chromogenic cephalosporin assay was performed in microtitre trays, and a positive result was indicated by the development of a red colour within 30 minutes. The disc diffusion assay was performed using modified Thayer-Martin medium, and an inhibition zone size of less than $20 \mathrm{~mm}$ diameter was regarded as indicating a positive result.

With all the methods it was necessary to use a heavy inoculum to achieve the best results.

\section{Results}

Of a total of 202 strains tested, 99 were found to be PPNG strains. The rapid iodometric, chromogenic cephalosporin, and disc diffusion assays gave complete agreement with all the strains (table I). The filter paper iodometric method detected $99 \%$ of the PPNG strains. No false-positive result occurred with the filter paper method. Except for two strains which gave weakly positive results, the rest of the PPNG strains gave strongly positive results. With the filter paper technique, it was important to read the results immediately after the addition of the iodine, because the paper became decolorised after about 30 seconds.

TABLE I Detection of penicillinase-producing Neisseria gonorrhoeae by different methods

\begin{tabular}{lll}
\hline & \multicolumn{2}{l}{ No of strains } \\
\cline { 2 - 3 } Methods & + & - \\
\hline Rapid iodometric & 99 & 103 \\
Chromogenic cephalosporin & 99 & 103 \\
Disc diffusion & 99 & 103 \\
Paper iodometric & 98 & 104 \\
\hline + Positive - negative & &
\end{tabular}

Both the rapid iodometric and chromogenic methods gave unequivocal results with all the strains. In a separate study $96 \%$ of 50 PPNG strains decolorised the starch in the rapid iodometric test after only 10 minutes' incubation of the organisms with the penicillin solution. One strain required $\mathbf{3 0}$ minutes' and another 60 minutes' incubation time. The latter strain also gave a false-negative result with the paper iodometric method.

All the PPNG strains except one turned the cephalosporin $87 / 312$ red within two minutes of the organisms being mixed with the antibiotic. For the strain which gave a false-negative result with the filter paper iodometric method the cephalosporin took 15 minutes to turn red.

The inhibition zone sizes of the strains, using the disc diffusion method, are shown in table II. None of the PPNG strains, except one, showed any zone of inhibition around the penicillin disc. The strain which gave a negative result with the filter paper iodometric method had a zone of inhibition $16 \mathrm{~mm}$ in diameter. All the non-PPNG strains had zone sizes of $22 \mathrm{~mm}$ or more.

TABLE II Sizes of penicillin inhibition zones of Neisseria gonorrhoeae strains

\begin{tabular}{lcc}
\hline Strains & Size in $\mathrm{mm}$ & No \\
\hline PPNG & 0 & 98 \\
Non-PPNG & 16 & 1 \\
& $22 \& 23$ & 5 \\
& $24 \& 25$ & 18 \\
& $26 \& 27$ & 23 \\
& $28 \& 29$ & 20 \\
& $\geqslant 30$ & 37 \\
\hline
\end{tabular}

\section{Discussion}

The detection of penicillinase-producing $N$ gonorrhoeae strains requires isolation of the gonococcus. It is desirable that the method adopted by laboratories for detecting $\beta$-lactamase-production should be reliable, simple, rapid, and cheap. However, most of the current methods are fairly labour-intensive, and the disc diffusion method requires an additional day before results are known. The chromogenic cephalosporin method, being specific, sensitive, simple, and rapid, comes closest to satisfying optimum requirements. Unfortunately the reagent is not readily available and most laboratories will have to use one or more of the other methods.

In localities where the prevalence of PPNG strains is low, it is probably unnecessary to test all gonococcal isolates for $\beta$-lactamase-production routinely. It is sufficient to test strains from patients in whom treatment with penicillin has failed or who have acquired their infections from PPNG-endemic areas. For laboratories in such areas, the disc diffusion method is practical since the materials necessary for performing the test are readily available. The present study shows that there was complete agreement between the results of the disc diffusion test and the rapid iodometric or chromogenic cephalosporin tests. Even though a high proportion of the nonPPNG strains in Singapore are relatively resistant to penicillin, there was no difficulty in distinguishing the PPNG from the non-PPNG strains using a 20-mm zone size as the criterion for separating the 
two groups. Only one PPNG strain had a zone of inhibition and it measured $16 \mathrm{~mm}$. The smallest zone size for the non-PPNG strain was $22 \mathrm{~mm}$. Strains which are suspected of being PPNG should be confirmed by either the chromogenic cephalosporin or rapid iodometric methods.

Where the prevalence of PPNG strains is about $1 \%$ or more, it is likely that the treatment failure rate with penicillin will be high. In such localities it would be prudent to screen all gonococcal isolates for $\beta$-lactamase. In laboratories with a low turnover, either the chromogenic cephalosporin or rapid iodometric method is suitable. The results of the two tests showed complete agreement and were unequivocal. The reaction in the chromogenic cephalosporin assay was very rapid. A red coloration appeared within two minutes for all the PPNG stains except one; in this case, it appeared within 15 minutes. The breakdown of penicillin in the rapid iodometric method was also fast since $96 \%$ of 50 PPNG strains (in a separate study) required only 10 minutes' incubation time. However, since one strain required 30 minutes' and another 60 minutes' incubation time, it is important to adhere to the latter incubation time.

Where there is a high turnover, laboratories might consider using the simpler filter paper iodometric method. The test however requires some experience to obtain reproducible results. The original concentration of penicillin used, $100000 \mu \mathrm{g} / \mathrm{ml}$, is too strong, ${ }^{3}$ and more specific results are obtained with $60000 \mu \mathrm{g} / \mathrm{ml}$. The iodine solution is used at $0.5 \%$ concentration. It is also important that the starchimpregnated filter paper should not be kept for longer than a month, as deterioration of the starch may also give rise to false-positive results. The principle of the method is similar to the test described by Jorgensen et $a^{2}$ who in addition incorporated penicillin into the starch paper and were able to keep the test paper at $-20^{\circ} \mathrm{C}$ for a year. The prevalence of PPNG strains in Singapore was $0.2 \%$ in 1977 but rose to $10.1 \%$ in 1978 . The prevalence for the first 10 months of 1979 is $18.5 \%$. With such a high prevalence it has been necessary to screen all gonococcal isolates for $\beta$-lactamase. The simplicity of the filter paper method makes it possible to screen an average of 30 isolates a day. All positive results are confirmed by either the chromogenic cephalosporin or rapid iodometric method.

We thank Glaxo Research Ltd, England, for the compound $87 / 312$.

\section{References}

1. Catlin BW. Iodometric detection of Haemophilus influenzae $\beta$-lactamase: rapid presumptive test for ampicillin resistance. Antimicrob Agents Chemother 1975; 7:265-70.

2. Jorgensen JH, Lee JC, Alexander GA. Rapid penicillinase paper strip test for detection of $\beta$-lactamase-producing Haemophilus influenzae and Neisseria gonorrhoeae. Antimicrob Agents Chemother 1977;11:1087-8.

3. Odugbemi TO, Hafiz S, McEntegart MG. Penicillinaseproducing Neisseria gonorrhoeae: detection by starch paper technique. Br Med J 1977; ii: 500.

4. Thornsberry C, Kirven LA. Ampicillin resistance in Haemophilus influenzae as determined by a rapid test for $\beta$-lactamase production. Antimicrob Agents Chemother 1974;6:653-4.

5. Weissfeld AS, Sanner GD, Childress JR, et al. Feasibility of screening for penicillinase-producing Neisseria gonorrhoeae from primary culture plates by using a rapid microacidometric test. Antimicrob Agents Chemother 1977; 12:703-6.

6. O'Callaghan CH, Morris A, Kirby SM, et al. Novel method for detection of $\beta$-lactamase by using a chromogenic cephalosporin substrate. Antimicrob Agents Chemother 1972;1:283-8.

7. World Health Organisation. Neisseria gonorrhoeae producing penicillinase. WHO Wkly Epidem Rec 1976;38:293-4.

8. Hodge W, Ciak J, Tramont EC. Simple method for detection of penicillinase-producing Neisseria gonorrhoeae. J Clin Microbiol 1978; 7:102-3.

9. Martin JE Jr, Lewis JS. Selective culture screening for penicillinase-producing Neisseria gonorrhoeae. Lancet 1977; 2:605-6.

10. World Health Organisation. Techniques for the detection of $\beta$-lactamase-producing strains of $N$ gonorrhoeae. WHO Tech Rep Ser 1978;616: 137-42. 